UCRL-JC-128903

PREPRINT

\title{
Sheath Over a Finely Structured Divertor Plate
}

\author{
R.H. Cohen \\ R. Cid \\ E.B. Hooper \\ A.W. Molvik \\ G.D. Porter \\ D.D. Ryutov
}

This paper was prepared for submittal to

13th Intl. Conference on Plasma Surface Interactions in Controlled Fusion Devices

San Diego, CA

May 18-22, 1998

May 15, 1998

This is a preprint of a paper intended for publication in a journal or proceedings. Since changes may be made before publication, this preprint is made available with the understanding that it will not be cited or reproduced without the permission of the author. 


\section{DISCLAIMEIR}

This document was prepared as an account of work sponsored by an agency of the United States Government. Neither the United States Government nor the University of California nor any of their employees, makes any warranty, express or implied, or assumes any legal liability or responsibility for the accuracy, completeness, or usefulness of any information, apparatus, product, or process disclosed, or represents that its use would not infringe privately owned rights. Reference herein to any specific commercial product, process, or service by trade name, trademark, manufacturer, or otherwise, does not necessarily constitute or imply its endorsement, recommendation, or favoring by the United States Government or the University of California. The views and opinions of authors expressed herein do not necessarily state or reflect those of the United States Government or the University of California, and shall not be used for advertising or product endorsement purposes 


\title{
Sheath over a finely structured divertor plate
}

\author{
R.H. Cohen, R.Cid', E.B. Hooper, A.W. Molvik, G.D. Porter, and D.D. Ryutov \\ Lawrence Livermore National Laboratory, Livermore, CA 94551, USA
}

\section{Introduction}

The surface of a divertor plate typically has fine structure. Depending on the material and the duration of exposure to the plasma, the characteristic size of the surface imperfections may vary over a broad range. In this paper, we consider the case where these structures have scale $h$ that is much smaller than the ion gyroradius $\rho_{i}$ but greater than the electron gyroradius $\rho_{e}$ :

$$
\rho_{e}<h<\rho_{i}
$$

The magnetic field intersects the divertor plate at a shallow angle $\alpha<<1$. The present paper demonstrates that the combination of these two factors, fine surface structures and strongly tilted magnetic field, gives rise to many interesting new phenomena in the sheath (the case of smooth waviness of the surface was considered in Ref. [1]; the case of a rough surface with the features with sizes comparable with the ion gyroradius or greater was considered in Ref. [2]). We consider only the plasma part of the problem: given the presence of some structure, what are the consequences in terms of the plasma properties in the vicinity of the surface? We are not addressing the issue of what process has caused the appearance of the structure. However, once the plasma part of the problem is solved, on could return to the analysis of the wall erosion problem, based on the solution obtained. The analysis of the sputtering process for a finely structured surface was performed in Ref. [3].

For the environment of the divertor region of a medium-size tokamak (plasma density $n \sim 4 \cdot 10^{13} \mathrm{~cm}^{-3}$, plasma temperature $T \sim 50 \mathrm{eV}$, the magnetic field strength $B \sim 2 \mathrm{~T}$ ), one has: $\rho_{i}$ $\sim 500 \mu \mathrm{m}$ (hydrogen), $\rho_{e} \sim 10 \mu \mathrm{m}$. We, therefore, are going to analyze the scales of imperfections in the range $10 \mu \mathrm{m}<h<500 \mu \mathrm{m}$. These scales are quite typical for a number of fusion devices [4]. For reference purpose, one can mention that the Debye radius $\rho_{D}$ is $\sim 10$ $\mu \mathrm{m}$.

We will use the following model of the surface: we assume that it is formed by the randomly distributed cones of height $h$, with base radius and the distance between neighboring cones both also of order $h$ (Fig. 1). This latter assumption means that the number of cones per unit surface area is $\sim 1 h^{2}$. With minor modifications, the analysis can be extended to bumps

\footnotetext{
' Deprtment of Applied Science, University of California, Davis, CA 95616, USA
} 
with smooth tops (an example of such an analysis for $h>\rho_{i}$ can be found in Ref. 2). The approach used in this paper is based on scaling arguments and order-of magnitude estimates. Despite its simplicity, it allows one to obtain basic scaling relations and reveal some interesting phenomena.

After presenting a theoretical analysis of the plasma interaction with a finely structured surface, we present a brief description of a small-scale dedicated device for experimental studies of this problem.

\section{Ion absorption}

We will discuss the problem in two steps: first assume that electric field does not have a significant effect on the particle motion, and then consider possible complications. We first consider the case of very small $h$ 's, when the magnetic field doesn't directly affect the ion trajectories at a distance $\sim h$ from the wall. Even in this case, however, the presence of the magnetic field is important in that it causes the ions to approach the wall at a very shallow angle $\sim \alpha^{1 / 2}$ (we are talking of a "typical" ion, with a pitch-angle $\sim 1$ ). This is so because the ion approaching the wall along a strongly tilted magnetic field, when making one full gyrocircle, moves closer to the wall by only a small distance $\sim \alpha p_{i}$. The ion velocity components near the wall are: $\mathrm{v}_{\mathrm{x}} \sim \mathrm{v}_{\mathrm{z}} \sim \mathrm{v}_{\mathrm{Ti}}, \mathrm{v}_{\mathrm{y}} \sim \mathrm{v}_{\mathrm{Ti}} \alpha^{1 / 2}$ (see Fig. 1 for the definition of the coordinate axes). The details of the corresponding analysis and the full ion distribution function near the wall can be found in Ref. 5. The projection of the typical ion trajectory on the divertor plate forms an angle $\sim 45^{\circ}$ with the projection of the magnetic field.

So, we consider a stream of ions with a density $n$ moving with a velocity $\mathrm{v}$ along straight trajectories forming some small angle $\beta$ with the plane. The problem is: how far below the level of the mountain tops will the ions penetrate? This is a problem similar to the problem of a mountain range during sunrise: what fraction of the mountain land is illuminated? Lct us denote by $\Delta h$ the corresponding distance from the mountain tops (here and below we mean by $\Delta h$ a characteristic, statistically averaged quantity). The surface area of the illuminated mountain top is $\sim \Delta h^{2}$. Each mountain top collects $n v \Delta h^{2}$ ions per unit time. As the number of the mountain tops per unit area of the mountain land is $\sim 1 / h^{2}$, the number of ions absorbed by the unit area of the plate per unit time is $\sim n v \Delta h^{2} h^{2}$. On the other hand the number of the ions intersecting a unit surface element parallel to the plate at some distance from the plate is $\beta n \mathrm{v}$. Equating the two expressions, we obtain:

$$
\Delta h \sim \beta^{1 / 2} h
$$

As we have mentioned above, in the problem under consideration $\beta \sim \alpha^{1 / 2}$. Therefore,

$$
\Delta h \sim \alpha^{1 / 4} h
$$

Accordingly, the fraction $\varepsilon_{i}$ of the surface wetted by the ions is: 


$$
\varepsilon_{i} \sim \alpha^{1 / 2}
$$

Illuminated are south-east or north-east sides of the "mountains" (where field lines from the plasma in the sunrise analogy are assumed to intersect the east side), depending on whether the magnetic field is directed toward or away from the plate. Note, Eq. (4) remains formally valid even for infinitesimally small bumps (the constraints will be discussed in Sec. 3).

The estimate (3) is valid so long as the height $\Delta h$ is smaller than the distance $\alpha \rho_{i}$ by which the ion gyro-circle "descends" to the wall during one gyro-period (if $\Delta h$ becomes formally larger, the ion motion at the scales of interest begins to be affected by the magnetic field). Therefore, the applicability condition for the estimates (3), (4) reads:

$$
h<\rho_{i} \alpha^{3 / 4}
$$

When it breaks down, the following approach [2] can be used. From the kinematics of the gyro-motion, it is clear that the characteristic vertical velocity of the ions whose gyrocenters have lowered by a distance $\Delta h$ since the moment when the gyro-circle has just touched the mountain tops (Fig. 2), is $\mathrm{v}_{\mathrm{Ti}}\left(\Delta h / \rho_{i}\right)^{1 / 2}$. The other two velocity components are of the order of $v_{\mathrm{Ti}}$. The phase-space density of ions moving in a magnetic field is constant and, therefore, their volume density is proportional to the volume occupied in the velocity space. At distances exceeding $\rho_{i}+h$ from the wall, this volume is $\sim \mathrm{v}^{3} \mathrm{~T}$, whereas in the layer of thickness $\Delta h$ containing the mountain tops it is $\sim\left(\Delta h / \rho_{i}\right)^{1 / 2} \mathrm{v}^{3} \mathrm{Ti}$. Accordingly, in the layer of thickness $\Delta h$, their density is $\sim n\left(\Delta h / \rho_{i}\right)^{1 / 2}$. The number of ions absorbed by one mountain top per unit time is $\sim \Delta h^{2}\left(\Delta h / \rho_{i}\right)^{l / 2} \mathrm{v}_{\mathrm{Ti}}$, and the number of ions absorbed per unit area of the divertor plate per unit time is $\sim(\Delta h / h)^{2}\left(\Delta h / \rho_{i}\right)^{1 / 2} \mathrm{v}_{\mathrm{Ti}}$. On the other hand, the flux of ions approaching the divertor plate from the plasma is $\alpha n v_{T i}$ (per unit surface). Equating the two quantities, one finds that

$$
\Delta h / h \sim \alpha^{2 / 5}\left(\rho_{i} / h\right)^{1 / 5}
$$

The fraction of the surface wetted by the ions is:

$$
\varepsilon_{i} \sim \alpha^{4 / 5}\left(\frac{\rho_{i}}{h}\right)^{2 / 5}
$$

The transition between the estimates (3) and (6) [and (4) and (7)] occurs at the point where inequality (5) becomes equality.

Equations (6) and (7) remain valid so long as the illuminated height $\Delta h$ remains smaller than $\rho_{i}$. This is so at

$$
h<\rho_{i} / \alpha^{1 / 2},
$$

i.e., even at heights exceeding the ion gyroradius (the result was derived in [2] under the stated assumption that $\left.\rho_{i}<h\right)$. At even greater heights, when this inequality breaks down, the ion gyrocircles become small compared with the size of the illuminated mountain top, and the description of the gyrocircles as point particles moving strictly along the magnetic field 
becomes possible. This latter case was studied in detail in Ref. 2. It corresponds to the case considered at the beginning of this section, but with $\beta=\alpha$. Accordingly, the fraction of the wetted surface was found to be:

$$
\varepsilon_{i} \sim \alpha
$$

\section{Electron absorption and electric field effects}

At equal electron and ion temperatures, the electron gyroradii are much smaller than the ion gyroradii. Therefore, in a number of cases of practical interest the electron absorption corresponds to the regime of an infinitesimal gyrocircle defined by inequality

$$
h>\rho_{e} / \alpha^{1 / 2}
$$

In this regime, the fraction of the surface accessible to the electrons is determined by expression (9). In some cases, the regime defined by the inequality opposite to (10) may be realized. In such a regime the fraction of the surface accessible for electrons is determined from the estimate (7), but with $\rho_{i}$ replaced by $\rho_{e}$. The division of the parameter space by these inequalities is shown in Fig. 3. Expressions for the fraction of the wetted surface are presented in the 'lable 1.

We have been so far ignoring the presence of the electric fields and the quasineutrality constraint. The first effect of this constraint is that the bulk plasma must be charged positively with respect to the wall, to provide equal electron and ion currents (for definiteness, we consider the situation where there is no current to the wall). The corresponding potential is of the order of (3-5)T/e. For

$$
\alpha>\sqrt{m_{e} / m_{i}}
$$

a considerable fraction of this potential drop occurs within the Debye sheath immediately adjacent to the wall, whereas the rest of the drop occurs in a smooth fashion at distances of the order of the ion gyroradius (see, e.g., [6]). We will assume that inequality (11) is satisfied.

The second effect of the quasineutrality constraint is that, as we see from Table 1 and Fig. 3 , in most regimes, if potentials are neglected, ions penetrate deeper beneath the mountain tops than the electrons (which are much more tightly tied to the magnetic field lines). Hence we see the second effect of the quasineutrality constraint: potentials must form to prevent ions from entering the spatial domain inaccessible for electrons in the shadows of the mountaintops. Therefore, with the quasineutrality constraint imposed, the are wetted by the ions will become equal to the area wetted by the electrons.

The transitional zone (that repels the ions) has a positive potential with respect to the walls. Therefore, if even a weak source of ionization acts on the corresponding field lines, the cold electrons produced there will be trapped electrostatically (whereas the ions will be expelled). The neutral atoms may come as a result of surface recombination of the impinging 
ions. The ionization of neutral particles will gradually allow the plasma ions to penetrate deeper and deeper into the zone initially inaccessible for them (a manifestation of the Simon short-circuit effect [6]) and, eventually, will restore the situation where the ions will be "wetting" a considerably greater surface than the electrons. It is interesting to note that, while the electrons "illuminate" the surface from the "east," the ions hit the cones from "south-east" or "north-east". This may cause the appearance of a characteristic pattern of evolution of the surface relief.

Electric field present in the ion sub-sheath may also cause some changes of the overall picture in the case of non-uniformities which are smaller than the ion gyro-radius. As was shown in [5], some ions (predominantly those with a small energy) may be accelerated to the walls from the middle of the ion sub-sheath, and approach the wall at an angle $\sim 1$. They will be wetting the surface almost uniformly. However, as was shown in [5], the majority of the thermal ions will still approach the wall in the same qualitative way as at no electric field at all. They will be wetting a small fraction of the surface determined by Eqs. (4) or (7).

When surface non-uniformities become smaller than the Debye radius, the ions entering the Debye sheath experience a strong acceleration in the normal direction to the wall and, because of this, approach the wall at an angle $\sim 1$. This restores uniform wetting of the surface by the ions and sets the applicability limit for the estimate (4). It is interesting to note that the plasma electrons in the case of the non-uniformities with $h<<\rho_{e}$ still reach the wall only near the mountain tops, and the fraction of the area wetted by the electrons follows Eq. (4) even for the infinitesimally small cones.

\section{Secondary electron emission.}

It is well known that the secondary emission coefficient for a flat surface in a strongly tilted magnetic field may become much smaller than for a magnetic field intersecting the wall at an angle $\sim 1$ (see, e.g., [6] and references therein). Consider as an example a problem where the electron gyroradius is considerably smaller than the Debye radius and one can, therefore, neglect the effects of a normal clectric ficld in the immcdiate vicinity of the surface. Then, the majority of the secondary electrons emitted from the wall will return to the wall because of their gyro-motion. Only those secondary electrons whose velocity is directed almost parallel to the magnetic field will reach the bulk plasma. An estimate of the secondary emission coefficient $S$ reads in this case:

$$
S \sim \alpha^{1 / 2} S_{0}
$$

(where $S_{0}$ is a secondary emission coefficient in the absence of the magnetic field, and the exact numerical factor depends on the angular distribution of the secondary electrons). In the case of a very strong magnetic field, where the electrons are strictly attached to field lines, 
transition to a rough surface would bring $S$ close to $S_{0}$, because the magnetic field intersects the surface of the cones at an angle $\sim 1$. At weaker magnetic fields, $S$ will tend to be smaller, because the surface wetted by relatively fast plasma electrons [satsifying condition (8)] would extend to a considerable distance beneath the mountain tips; hence the cold secondary electrons, which follow the fieid lines, will most often hit other mountain tops on their way from the surface.

\section{The Bluebell device.}

We are constructing a small experimental facility, "Bluebell", to explore the effects of surface roughness discussed here, as well as the larger-lateral-scale waviness discussed in Ref. [1]. The experiment uses a helicon plasma source [8] to create an argon plasma flow with ion kinetic energies in the range of a few eV. The plasma density is in the range of $2 \cdot 10^{12} \mathrm{~cm}^{-3}$, the axial magnetic field is in the range 100-200 G (in the zone of a free plasma stream outside the RF source region). The length of the free-flow zone is $50 \mathrm{~cm}$, the diameter of the vacuum chamber is $30 \mathrm{~cm}$. A $\sim 15-\mathrm{cm}$-long metal cone (whence the name of the device) with the axis parallel to the magnetic field and a small angle at the apex (between 5 and $10^{\circ}$ ) will be installed in the plasma stream. The axial symmetry of this surface creates a well-defined initial state; installing bumps of various size, dielectric impregnations, and other structures, one will be able to compare the resulting potential distribution in the incoming plasma flow with a reference case and thereby evalutate the effect of the surface non-uniformities. Provisions will be made for electric biasing of the elements of the surface. An alternative method of creating the required geometry is using a funnel-shaped magnetic field structure, with a metal cylinder (with the axis coinciding with the magnetic axis) installed in it. Again, an axisymmetric surface forming a small angle with the magnetic field will be created. The test elements will be installed on this surface. The cone (or the cylinder) will have translational (along the axis) and rotational (around the axis) degrees of freedom, allowing one to move the structured elements of the surface relative to the fixed arrays of Langmuir probes.

A special group of experiment directed towards studying the "shadowing" effect will be performed. The obstacles placed on the path of the flowing plasma would create shadows. If the size of the obstacles is much greater than the electron gyro-radius but much smaller than the ion gyro-radius, electrostatic barrier will be formed near the surface of the shadowed flux-tube, preventing the ions from entering into the shadow (in the same way as it was discussed in Sec. 3). The plasma density and potential distribution near this surface will be measured, and possible plasma instabilities (causeing anomalous penetration of the plasna into the shadows) will be studied. First plasma has been obtained in the device and experiments directed towards its characterization are underway. 


\section{Summary and discussion.}

We have examined the plasma properties near a bumpy wall which is nearly tangent to the impinging magnetic field. We see that for a wide range of feature heights, ranging from many ion gyroradii to of the order of the Debye length, only a small fraction of the surface is "wetted" by the main plasma electrons and ions. When the scale height is not large compared to the ion gyroradius, the electron and ion wetted fractions are significantly different (though both small). This gives rise to potentials that lead to a filling in of the ion-rich region with cold neutralizing electrons from ionization of gas. After this filling in, the residual differences in electron and ion loss channels, as well as temperature variations between the illuminated and shadow plasmas, will leave potential variations of the order of the electron temperature on a cross-field scale of the order of the illuminated portion of the surface bumps, $\Delta h$. These potential structures should extend a considerable distance back into the impinging plasma.

We are constructing a small dedicated experimental facility, the "Bluebell" device, to examine the phenomena discussed here as well as larger-lateral-scale surface waviness.

There are a number of consequences of the geometric relations and the shadowing that merit further exploration. One is secondary emission; as we noted above, a bumpy surface with scale height exceeding the electron gyroradius may not display the drop with angle of field-line incidence expected for a flat surface. Another question, which we plan to examine theoretically and in our experimental device, is whether a plasma boundary instability could also play a role in filling in the electron shadows.

There is also the challenge of closing the self-consistency loop to calculate the surface erosion. Certainly the restriction of the impinging plasma to the tips of the "mountains" and the different wetted areas as well as different directions of approach of electrons and ions will have significant impact on the erosion process. On the other hand, it is worth emphasizing that a strong concentration of the heat and particle flux near the mountaintops does not necessarily mean a fast self-healing of the surface imperfections. It should be remembered that there are many other processes playing important role in establishing a steady state surface relief: i) crack formation under the action of a cyclic heat load, with subsequent flaking; ii) cracking and blistering under the action of the absorbed neutral hydrogen; iii) blistering and flaking under the action of the neutron flux in the fusion reactor conditions; iv) damage from disruption events. The enhanced sputtering of the tops of the bumps is just one more factor that affects the evolution of the surface relief.

\section{Acknowledgment.}

This work was carried out under the auspices of the U.S. Department of Energy by Lawrence Livermore National I aboratory under Contract W-7405-ENG-48. 


\section{References}

1. R.H. Cohen, D.D. Ryutov, Nucl. Fusion, 37, 621, (1997).

2. R.H. Cohen, D.D. Ryutov, Physics of Plasmas, 5, \#6, (1998).

3. J.N. Brooks, D.N. Ruzic., Journal of Nuclear Materials, 176\&177, 278 (1990).

4. E. Deksnis, A. Cheetham, A. Hwang, P. Lomas, M. Pick, D.D.R. Summers. Journ. of Nucl. Mat., 176-177, 583 (1990); J. Linke, H. Bolt, R. Doerner et al. Journ. of Nucl. Mat., 176-177, 856 (1990); N. Yoshida, K. Tokunaga, T. Fujiwara, K. Tawara, T. Muroga, S. Itoh. Journ. of Nucl. Mat., 196-198, 415 (1992); T. Tanabe, V. Philipps, K. Nakamura et al. Journ. of Nucl. Mat., 241-243, 1164 (1997).

5. R.H. Cohen, D.D. Ryutov, Physics of Plasmas, 5, 808 (1998).

6. A. Simon. Phys. Rev. 75, 921 (1957).

7. S. Mizoshita, K. Shiraishi, N. Ohno, S. Takamura, J. Nucl. Mater., 220-222, 488 (1995).

8. A.V. Molvik et al. J. Vac. Sci. \& Technology, A14, 984 (1995). 
Table 1. Summary of wetted area vs. regime

\begin{tabular}{|c|c|c|}
\hline Domain & $\begin{array}{c}\text { Fraction of surface } \\
\text { wetted by ions }\end{array}$ & $\begin{array}{c}\text { Fraction of surface } \\
\text { wetted by electrons }\end{array}$ \\
\hline I & $\alpha$ & $\alpha$ \\
II & $\alpha^{4 / 5}(\rho \mathbf{j} / \mathrm{h})^{2 / 5}$ & $\alpha$ \\
III & $\alpha^{1 / 2}$ & $\alpha$ \\
IV & $\alpha^{4 / 5}(\rho \mathbf{j} / \mathrm{h})^{2 / 5}$ & $\alpha^{4 / 5}\left(\rho \mathrm{e}^{/ h}\right)^{2 / 5}$ \\
V & $\alpha^{1 / 2}$ & $\alpha^{4 / 5}\left(\rho_{\mathrm{e}} / \mathrm{h}\right)^{2 / 5}$ \\
\hline
\end{tabular}




\section{Figure captions.}

Fig. 1 A rough surface made of randomly distributed concs of the same height $h$. The arrow shows the direction of the magnetic field; in the divertor geometry, the axis $x$ corresponds to the radial, the axis $y$ to the poloidal, and the axis $z$ to the toroidal directions

Fig. 2 The ion gyro-circle viewed in the direction of the magnetic field; the solid lines

correspond to the instant when the lowest part of the circle is at the level of the mountaintops; the dashed lines correspond to some instant later in time, when the scrapingoff of the ions begins.

Fig. 3 The parameter domain of the problem. The lines correspond to deuterium. The line $\alpha=\left(\eta / \rho_{e}\right)^{4 / 3}$ is not shown because it does not fit the scale. 


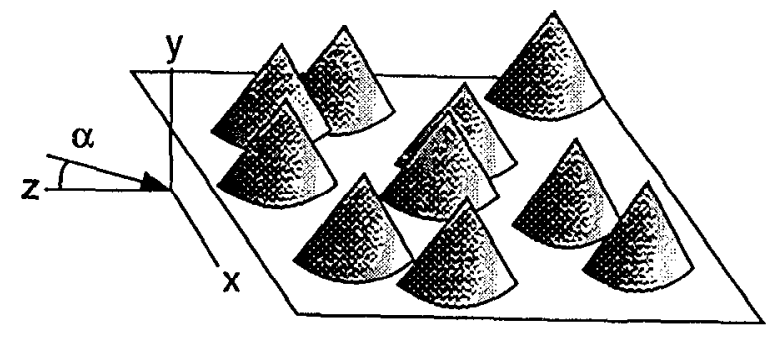

tig. 1 


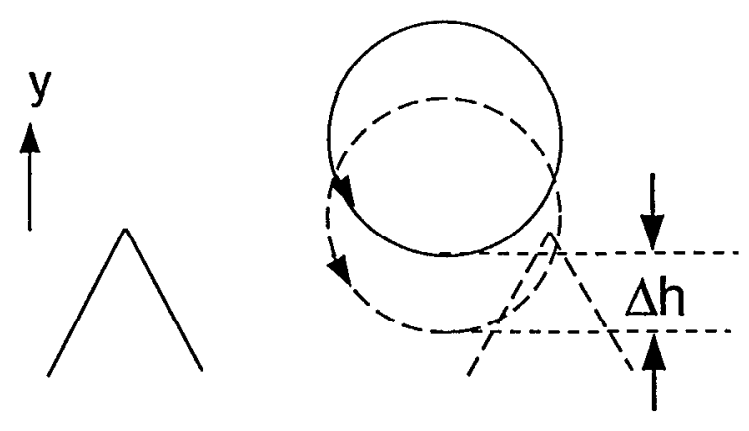

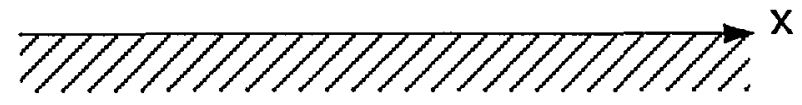




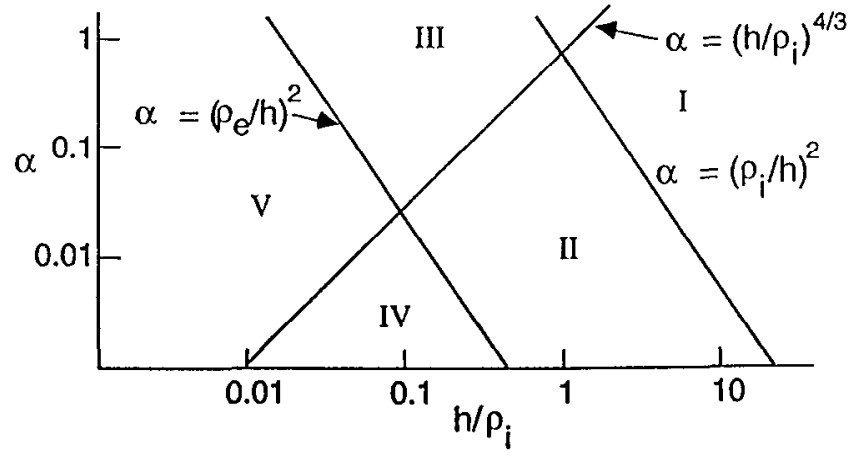

Fig. 3 


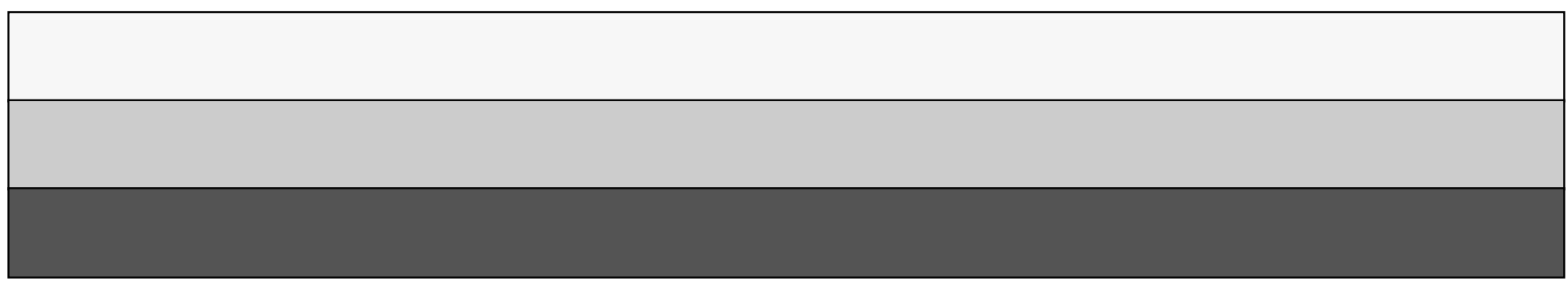

